\title{
Profiling of mitochondrial transcriptome in germinating wheat embryos and seedlings subjected to cold, salinity and osmotic stresses
}

\author{
Nayden G. Naydenov ${ }^{\dagger}$, Sakina Khanam ${ }^{\dagger}$, Maryna Siniauskaya and Chiharu Nakamura* \\ Laboratory of Plant Genetics, Department of Agroenvironmental Science, Graduate School of \\ Agricultural Science, Kobe University, Kobe 657-8501, Japan
}

(Received 10 August 2009, accepted 1 February 2010)

\begin{abstract}
Mitochondrial functions are potential targets of abiotic stresses that are major environmental factors limiting plant development and productivity. To evaluate mitochondrial responses to abiotic stresses we studied mitochondrial transcriptome profiles at the early stages of wheat development after imbibition under normal and induced stress conditions. Three stresses given were low temperature $\left(4^{\circ} \mathrm{C}\right)$, high salinity $(0.2 \mathrm{M} \mathrm{NaCl})$ and high osmotic potential $(0.3 \mathrm{M}$ mannitol $)$. All these stresses greatly reduced growth but dramatically increased respiration both via the cytochrome and alternative pathways. Macroarray analysis of the mitochondrial transcriptome revealed that most of the changes in transcript levels were stress specific but groups of genes responded commonly to different stresses. Under 3-days continuous stresses, 13 genes showed low temperature specific responses with either up- or down-regulation, while 14 and 23 genes showed responses specific to high salinity and high osmotic potential, respectively. On the other hand, 13 genes showed common responses, among which $c o b$ and $c c m F n$ increased their transcript levels while transcripts of the other genes including nad6, atp4 and atp9 decreased. The differential profiles of mitochondrial transcriptome revealed by the macroarray analysis were verified by the quantitative reverse transcriptase PCR analysis. Taken together, three among five nuclearencoded mitochondria-targeted genes included in the array showed decreases under the stresses, while $M n S O D$ and $A O X$ increased their transcript amounts. Our results indicated the existence of common and different regulatory mechanisms that can sense different abiotic stresses and modulate both nuclear and mitochondrial gene expression in germinating wheat embryos and seedlings.
\end{abstract}

Key words: abiotic stress, macroarray, mitochondrial biogenesis, transcriptome profiling, bread wheat (Triticum aestivum L.)

\section{INTRODUCTION}

Abiotic stresses such as low temperature, high salinity and high osmotic potential are the principal causes of crop failure, reducing average yields of most major crops by more than 50\% (Bray et al., 2000). To cope with these environmental stresses, plants execute a number of physiological and metabolic responses (Bohnert et al., 1995; Bray et al., 2000), which are regulated mostly at the gene expression level (Houde et al., 2006). Mitochondria can play a role to adjust and maintain cellular homeostasis and integrity in highly variable environments (Rachmilevitch et al., 2007). During germination and early seedling

\footnotetext{
Edited by Toru Terachi

* Corresponding author. E-mail: nakamura@kobe-u.ac.jp

$\dagger$ These authors contributed equally to this work
}

growth, which is one of the most critical stages of plant development, mitochondrial biogenesis results from de novo synthesis of mitochondrial constituents (Morohashi, 1986). Without functional oxidative phosphorylation, however, the energy cost for biosynthetic processes cannot be fulfilled to help mitochondria adjust to different abiotic stresses. Morphological and biochemical evidence suggests that seed mitochondria become functional at the onset of imbibition and their integrity and performance systematically improve during germination under normal conditions (Macherel et al., 2007; Naydenov et al., 2008).

The expression of numerous plant genes is regulated by environmental stresses (Thomashow, 1999; Bray et al., 2000; Shinozaki et al., 2003). Number of studies addressed in nuclear transcriptome, e. g. those in cold, drought and salt stressed Arabidopsis (Seki et al., 2002), 
water stressed maize seedlings (Zheng et al., 2004), sulfur stressed Arabidopsis (Hirai et al., 2003), cold, drought, salt and abscisic acid stressed rice (Rabbani et al., 2003), salt and drought stressed wheat (Bartoli et al., 2005; Kawaura et al., 2006), and salt and drought stressed barley (Ozturk et al., 2002; Talame et al., 2007). The extent of contribution of organellar gene expression in such stress response however remains largely unknown. A few studies have focused on mitochondrial gene expression, including ones in tobacco cultured cells during nutrient starvation (Giegé et al., 2005) and in rice plants germinating under low oxygen conditions (Howell et al., 2007). In rice, some mitochondrial components (cox2, $\left.\operatorname{cox} 5 b-2, F_{1} \beta-1, F_{1} \beta-2\right)$ respond to low oxygen availability at both transcript and protein levels, while others like nad9 and cob remain unaffected (Howell et al., 2007). In pea leaf mitochondria, various stresses imposed differential impact on mitochondrial function and stability of key matrix enzymes, with low temperature causing more damage than drought (Taylor et al., 2005). Giegé et al. (2005), on the other hand, suggested that mitochondrial gene expression in Arabidopsis was insensitive to the stress imposed by sugar starvation, but instead mitochondrial biogenesis was governed largely by changes in the nuclear gene expression through complex transcriptional and post-transcriptional events.

Mitochondrial respiration and biogenesis are possible targets of abiotic stresses especially during the process of germination when mitochondria are the sole energy producing organella. We herein report a study of mitochondrial gene expression profiles in germinating wheat embryos and seedlings subjected to cold, high salinity and high osmotic potential. Our aim was to study possible effect of cold, high salinity and osmotic stresses on mitochondrial development and function during germination and early seedling growth. For this we performed macroarray analysis using a set of 28 mitochondrial genes and 5 nuclear-encoded and mitochondria-targeted genes (Khanam et al., 2007). We pursued two particular objectives: 1) to identify mRNAs that change in abundance in a "stress-specific" fashion, and 2) to identify mRNAs that are co-regulated and exhibit a "shared stress" response during the early growth phase, i.e. for 3 days after germination. This is the first study to compare all three kinds of stresses using an expression profiling strategy in plant mitochondrial genome. Our results showed that changes in the gene expression level were mostly stressspecific.

\section{MATERIALS AND METHODS}

Plant materials and experimental conditions Germinating seeds of common wheat (Triticum aestivum L.) cultivar Chinese Spring were used in all experiments. Three kinds of stresses, i.e. low temperature, high salinity and high osmotic potential were applied to germinating embryos after imbibition and seedlings. Imbibition and sterilization of seeds were performed according to Khanam et al. (2007). For low temperature stress, imbibed seeds were placed on filter paper containing $0.1 \%$ Hyponex $(\mathrm{N}$ $\mathrm{P}-\mathrm{K}=5-10-5$, Hyponex, Osaka, Japan) solution in the dark at $4^{\circ} \mathrm{C}$ for 1 to 3 days. For salinity and osmotic stresses, the Hyponex solution was adjusted to $0.2 \mathrm{M}$ $\mathrm{NaCl}$ and $0.3 \mathrm{M}$ mannitol, respectively, and seeds were kept for 3 days in the dark at $25^{\circ} \mathrm{C}$. To study the effect of low temperature at different stages during the 3-days test period, seeds were kept at $4^{\circ} \mathrm{C}$ for 1 day after being incubated for 1 or 2 days under normal temperature at $25^{\circ} \mathrm{C}$. We designed these low temperature stress conditions to study the short term effect of low temperature during the initial stages of germination as in nature germinating wheat seeds frequently cope with this kind of stress especially in the temperate region where temperature suddenly drops in the night and then becomes normal during the day in early spring. After these stress treatments fresh weights and lengths of shoot and root tissues were measured. The whole experiment was repeated twice with ten samples per experiment under the dark condition to avoid any effects from photosynthesis.

Measurement of respiratory oxygen consumption A Clark-type oxygen electrode (Yellow Spring Instrument, USA) was used for the measurement of respiratory oxygen consumption in germinating embryos and developing seedlings. Before measurement, tissues were vacuum infiltrated with buffer solution (10 mM Mes-50 mM Hepes, $\mathrm{pH}$ 6.6) for 2-3 min to allow penetration of oxygen and respiratory inhibitors. The rate of oxygen consumption was measured in the dark by placing embryos and seedlings cut into ca. $2 \mathrm{~mm}$ ling tissues directly in $3 \mathrm{ml}$ of the buffer solution. The reactions were initiated by the addition of infiltrated embryos followed by KCN (2 $\mathrm{mM}$ final concentration) and $n$-propyl gallate (n-PG, 2 $\mathrm{mM}$ final concentration). Reactions were followed for a total of $10 \mathrm{~min}$ : KCN was added $1 \mathrm{~min}$ after initiating the reaction; $\mathrm{n}-\mathrm{PG}$ was added after $8 \mathrm{~min}$. The activity measured in this assay represents the $\mathrm{CN}$-resistant and $\mathrm{nPG}$ sensitive oxygen uptake by AOX. On the other hand the capacity of the cytochrome pathway was measured by reversing the order of addition of inhibitors and it represents $\mathrm{nPG}$-resistant and $\mathrm{CN}$-sensitive oxygen uptake by cytochrome oxidase. The residual (non-inhibited) respiration was subtracted from the measurements. The whole experiment was repeated twice with ten samples per experiment.

Macroarray analysis for mitochondrial gene expression For constructing primers in the macroarray analysis, we used sequence information of mitochondrial genomes of rice (Notsu et al., 2002) and wheat (Ogihara 
et al., 2005). All protein-coding genes of rice are commonly present in wheat except for rps19 and rpl2, which are functional in rice but not in wheat and thus were not included in this study. We thus selected 20 genes encoding proteins with known function and an open reading frame orf176 from the rice mitochondrial genome. In addition, 4 mitochondrial protein-coding genes (atp4, $a t p 8, c c m F c$ and $m t t B$ ), 2 ribosomal protein-coding genes (rps 7 and rps 12), and 2 open reading frames (orf349 and orf359) from wheat mitochondrial genome were included. We also selected 4 nuclear encoded and mitochondriatargeted wheat genes (Whlp, TaMRPL5, TaMRPL11 and $M n-S O D)$ plus wheat Ubiquitin and Actin genes as controls. Primer sequences, preparation of membrane filters and all other steps including RNA isolation, reverse transcription, hybridization, washing, signal detection and data analysis were as described in Khanam et al. (2007). RNA labeling was repeated twice using independently isolated total RNA samples, and hybridization was carried out twice with independently prepared membrane filters.

Signal intensities were determined by scanning X-ray films (Fuji) using the acquisition software Image $J$ (available at http//rsb.info.nih.gov/ij). For each gene, signal intensities of two spots were averaged and the mean was normalized with respect to the intensity of wheat actin gene (gi:48927617) on the same membrane. Signal intensities from two independent arrays were further averaged and used to calculate the changes in gene expression levels. Student's t-test was used for determining significance in gene expression levels with $\mathrm{p}$ value $<0.05$. Transcripts that could not be detected under stressed conditions but were readily detected in corresponding normal conditions were arbitrary scored as ones with more than 3-fold decrease in their expression. The differentially expressed genes were clustered using pairwise single-linkage hierarchical clustering based on Pearson correlation: Cluster 3.0 (de Hoon et al., 2004). Heat maps were generated using Java Treeview (Saldanha, 2004).

Quantitative RT-PCR analysis To validate the results obtained by the macroarray analyses, quantitative reverse transcriptase PCR (qRT-PCR) was performed using the Light Cycler instrument with the Light Cycler ${ }^{\circledR}$

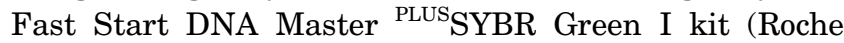
Diagnostics) on a selected set of representative genes with varying expression profiles. The mitochondrial genes of wheat analyzed with qRT-PCR were nad7 (complex I), cob (complex III), cox2 (complex IV) and atp6 (complex V) and rps 7 in addition to three nuclear-encoded mitochondriatargeted wheat genes, $M n S O D$ for manganese superoxide dismutase, TamRPL5 for mitchondrial ribosomal protein (Mizuno et al., 2008) and Waox1a for alternative oxidase (Takumi et al., 2002). Primer sequences and qRT-PCR condition were as described previously (Naydenov et al., 2008). The whole experiment was repeated twice using independently isolated total RNA. Relative expression of each target gene was calculated by the $\Delta \Delta \mathrm{C}_{\mathrm{T}}$ method, where wheat actin gene expression was used as reference (Livak and Schimittgen, 2001).

\section{RESULTS}

Embryo germination and shoot and root growth under stress conditions Embryo germination and seedling growth are inhibited by high salinity and osmotic stresses in durum wheat (Almansouri et al., 2001) and in rice (Kawasaki et al., 2001). Incubation of imbibed seeds at $4^{\circ} \mathrm{C}$ for 1 day did not lead to any visible coleoptile protrusion but embryo fresh weight increased from 0.6 to 1.2 $\mathrm{mg}$ in average (Fig. 1A). This was presumably due to rehydration rather than a result of active growth and increase in shoot biomass. Continuous incubation at $4^{\circ} \mathrm{C}$ for 2 days led to visible coleoptile protrusion and a total weight of the emerging shoot increased to $2.0 \mathrm{mg}$. After 3-days incubation at $4^{\circ} \mathrm{C}$, the shoot weight increased further to reach $5.0 \mathrm{mg}$. During this period shoot weight increase steadily to ca. $50 \mathrm{mg}$ at $25^{\circ} \mathrm{C}$. The results indicated that although severely inhibited wheat metabolism did not stop and allowed for a detectable biomass increase under the low temperature stress. To determine the period in which germinating embryos are most susceptible to the low temperature stress, 1-day stress was applied during the second or the third day (see M \& M). At the end of this treatment shoot and root weights were measured and compared to those under normal conditions for the same period (Fig. 1B). On the 2nd day of stress, shoot growth was inhibited by $82 \%$, while on the 3rd day the rate of inhibition was $55 \%$. Root growth was similarly inhibited by low temperature, suggesting that the earlier stages were more sensitive to low temperature than the later stages.

For salinity and high osmotic potential stresses, we first determined the concentrations of $\mathrm{NaCl}$ and mannitol required for causing significant growth reduction. After checking a range of concentrations $(0.2$ to $0.5 \mathrm{M})$, we adopted $0.2 \mathrm{M}$ for $\mathrm{NaCl}$ and $0.3 \mathrm{M}$ for mannitol because these concentrations caused inhibition of shoot growth by more than $50 \%$ as compared to the control. No reduction was observed in germination rate but growth of germinating embryos and seedlings was inhibited by $87 \%$ by $0.2 \mathrm{M}$ $\mathrm{NaCl}$ and $77 \%$ by $0.3 \mathrm{M}$ mannitol when these stresses were given for continuous 3-days (Fig. 1C). Shoot and root length was also reduced greatly by $\mathrm{NaCl}$, while high osmotic potential stress did not cause as much reduction (data not shown). The results showed that all three types of stresses significantly decreased wheat seedling growth as compared to the normal conditions.

Development of respiration under stress conditions We estimated the effects of stresses on mitochon- 
A

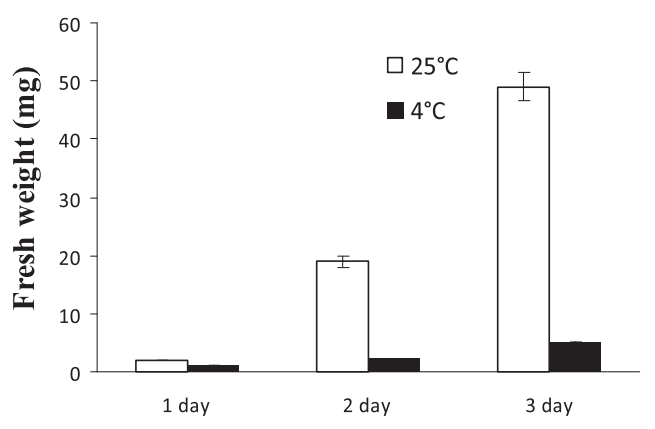

C

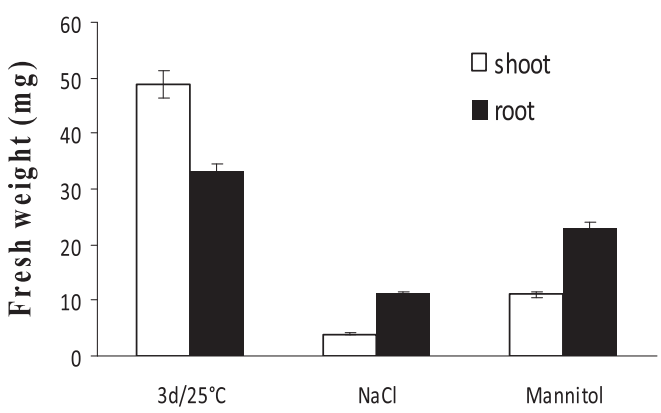

B

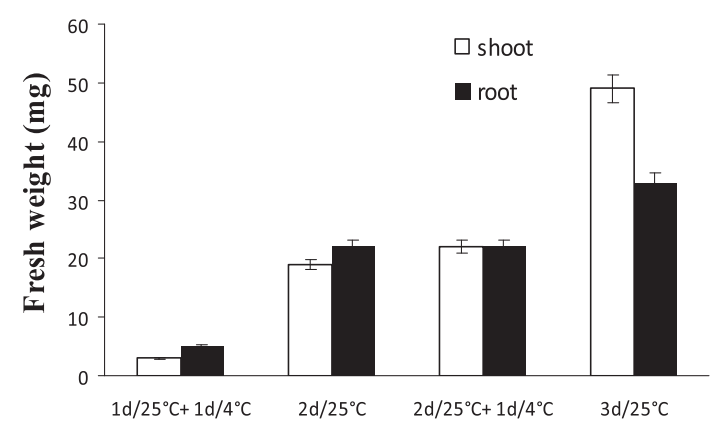

Fig. 1. Embryo development and seedling growth under different abiotic stress conditions. (A) Fresh weight of imbibed embryos and germinating seedlings under normal and low temperature for 1 to 3 days after imbibition. (B) Shoot and root fresh weight of seedlings incubated under different stress conditions. $1 \mathrm{~d} / 25^{\circ} \mathrm{C}+1 \mathrm{~d} / 4^{\circ} \mathrm{C}$, for example, represents a condition of 1 -day at $25^{\circ} \mathrm{C}$ followed by 1 day at $4^{\circ} \mathrm{C}$. (C) Shoot and root fresh weight of seedlings incubated for 3 days under normal temperature without $\left(3 \mathrm{~d} / 25^{\circ} \mathrm{C}\right)$ and with high salinity $(3 \mathrm{~d} / \mathrm{NaCl})$ and high osmotic potential (3d/Mannitol). Each bar represents a mean \pm standard deviation.

drial respiration in the initial phases of wheat growth after imbibition because mitochondrial respiration is the only energy supplying process until functional chloroplasts are formed to initiate photosynthesis. When imbibed seeds were subjected to the low temperature stress for 1 day, total respiration increased by $66 \%$ from the control, with $38 \%$ increase in the alternative pathway capacity and $43 \%$ in the cytochrome pathway capacity (Fig. 2A). Similar increases in both respiratory pathways as well as in the total respiration were observed when germinating embryos were subjected to the low temperature stress for 1 day during the second day (Fig. 2B). Even greater increase was observed when the low temperature stress was applied for continuous 2 days and this increase was attributed essentially to an increase in the cytochrome pathway, while the capacity of the alternative pathway decreased by $14 \%$ as compared to the control condition (Fig. 2B). When the low temperature stress was applied for 1 day during the 3 rd day the total respiration increased by $58 \%$, with increases in the cytochrome and alternative pathways of $20 \%$ and $40 \%$, respectively, as compared to the control condition (Fig. 2C). Continuous low temperature stress for 3 days led to nearly a four-fold increase in the total respiration with more than a five-fold increase in the cytochrome pathway. These data suggested that low temperature required wheat seedlings to respire at higher rates, possibly because elevated electron and solute leakage was compensated for by the increased respiration rate.

We next evaluated the energy demand of seedlings incubated under salinity and high osmotic potential stresses by monitoring the development of respiration. Under the normal temperature condition $\left(25^{\circ} \mathrm{C}\right.$, dark $)$ the cytochrome pathway capacity was approximately two-fold higher than the alternative pathway capacity at the end of third day after imbibition (Fig. 2D). Under $0.2 \mathrm{M}$ $\mathrm{NaCl}$ and $0.3 \mathrm{M}$ mannitol high osmotic potential stress conditions the total oxygen consumption increased by 2.7 and 2.4-fold, respectively, as compared to the normal condition. Greater increases occurred in the respiration via the alternative pathway than the cytochrome pathway, indicating that wheat seedlings responded differently to the high salinity and high osmotic potential stresses as compared to the low temperature stress. Importantly, stress-induced elevation of respiration was the greatest at the time of the greatest growth depression. This negative relationship agrees with the observation that respiration was higher in plants whose growth was 
A

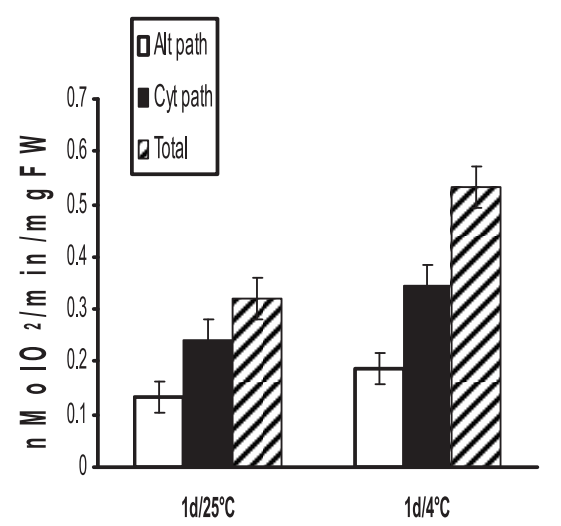

C

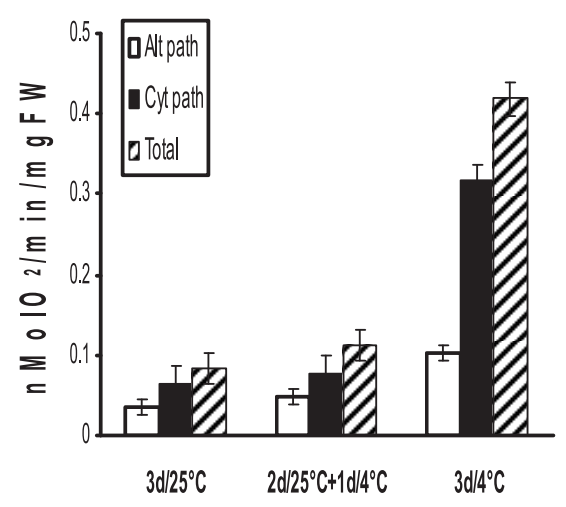

B

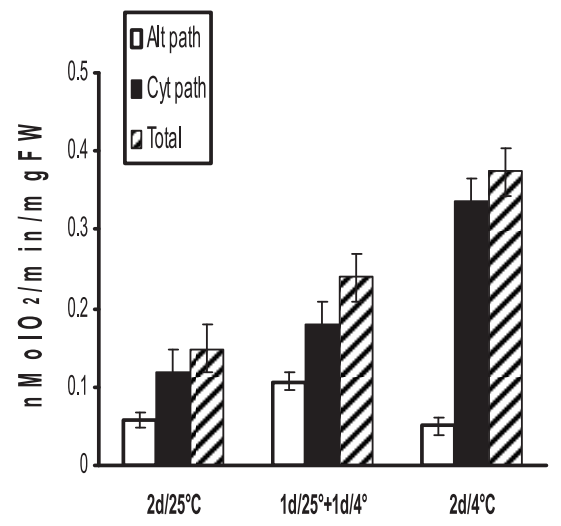

D

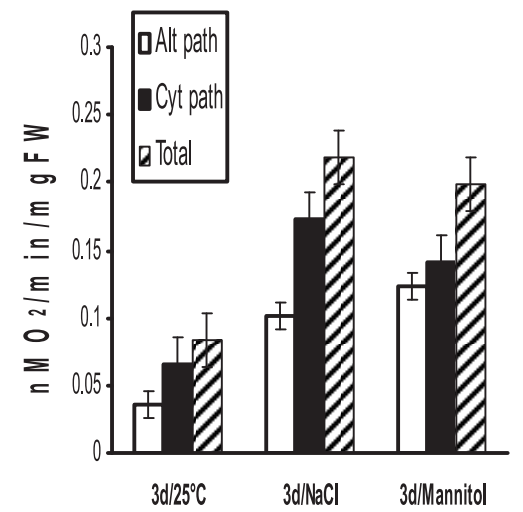

Fig. 2. Total respiration and capacity of the cytochrome and alternative pathways in germinating embryos and seedlings under different abiotic stress conditions. For the stress conditions, see the legends to Fig. 1. Each bar represents a mean \pm standard deviation.

highly depressed under extended $\mathrm{NaCl}$ stress (Nieman, 1962; Livne and Livne, 1967). The onset of respiratory increase in shoots paralleled the decrease in growth rate under the stresses, indicating changes in metabolic pathways. Among the three types of stresses tested, low temperature caused the highest respiratory increase and the higher increase was associated with prolonged treatment.

Macroarray analysis of mitochondrial gene expression under stress conditions We previously developed a macroarray system to study mitochondrial biogenesis by monitoring the level of mitochondrial transcripts in developing wheat seedlings under normal growth conditions (Khanam et al., 2007). We now attempted to apply this system to the stress conditions. We observed that under all three kinds of abiotic stresses shoot growth was inhibited more than root growth (Fig. 1), indicating that shoot tissues are more sensitive than root tissues to these stresses. We therefore used shoot cDNA in our mito- chondrial gene profiling experiment as we considered that the effects of stresses could be more pronounced in the shoot tissues.

To test the reproducibility of our array system, all measurements were repeated twice in completely independent experiments. High correlation coefficients were observed between the duplications in all conditions studied $(r=0.87-0.98)$, indicating high reproducibility of our macroarray system. For comparison between two different conditions, Fig. 3 shows, as an example, a scatter plot of genes with varying levels of signal intensities when seedlings incubated for 3 days under normal temperature were compared with those incubated for the same period under low temperature. In this case, many genes marked by squares and circles showed bigger than the 1.5-fold changes (arbitrarily chosen) between the two samples.

Using hierarchical cluster analysis, we attempted to find similarities and differences of the gene expression patterns and levels as wheat mitochondria responded to 


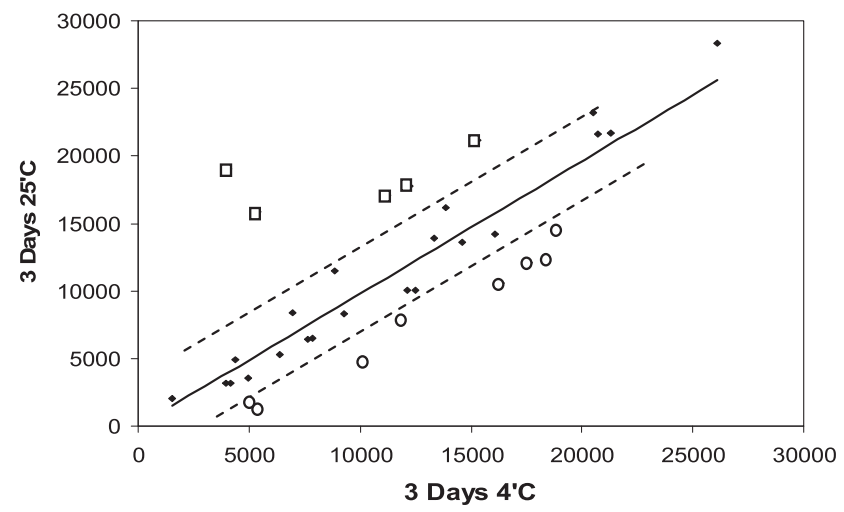

Fig. 3. Scatter plots of the signal intensities in the macroarray analysis. A comparison between arrays from the stress conditions $\left(3 \mathrm{~d} / 4^{\circ} \mathrm{C}\right)$ versus normal condition $\left(3 \mathrm{~d} / 25^{\circ} \mathrm{C}\right)$. Squares and circles represent outliers beyond the $+/-1.5$ fold changes. High correlation coefficients between duplications in all conditions studied $(r=0.87-0.98)$ indicated high reproducibility of the macroarray system. the three kinds of stresses. Gene clustering depended on fold changes in the abundance of each transcript under a given stress condition relative to the untreated control for the same period. In Fig. 4A were compared the transcript profiles in germinating embryos and seedlings incubated under continuous low temperature at $4^{\circ} \mathrm{C}$ for 1,2 and 3 days after germination with those under the control condition. At the end of the first day low temperature stress, 24 out of 33 genes showed decreases in their transcript levels, among which 8 genes (marked with asterisk) showed more than 3 -fold decreases compared to the control. After the 2-days and the 3-days stresses, 4 and 8 genes showed decreases in their transcript levels, respectively. On the other hand, 10 genes showed increases in their transcript abundance either after the 2 or 3-days of low temperature stress. These included respiratory subunitsencoding nad2 and nad5 (complex I), cob (complex III) and atp1 and atp4 (complex V) (clusters 1 and 2 in Fig. 4A). Only two genes, i.e. $c c m F n$ encoding translocase protein and $M n S O D$, were constantly up-regulated during the 3-days stress, although they did not show any
A

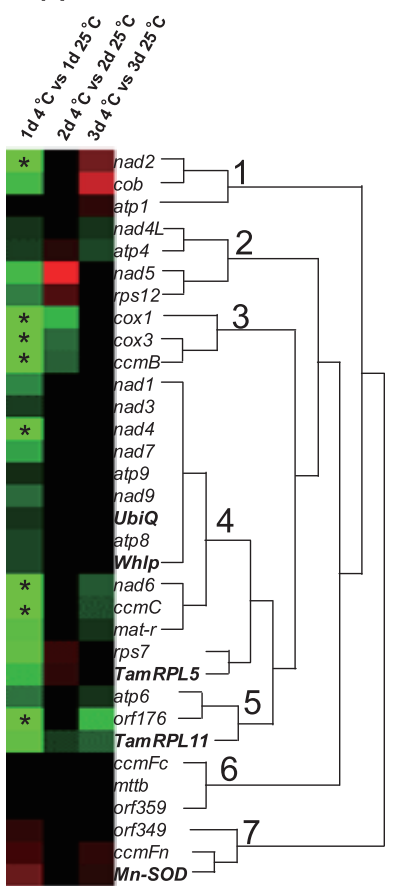

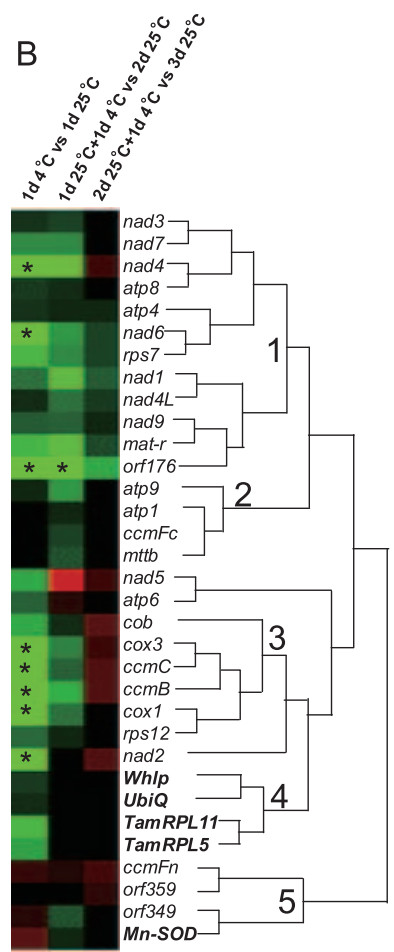

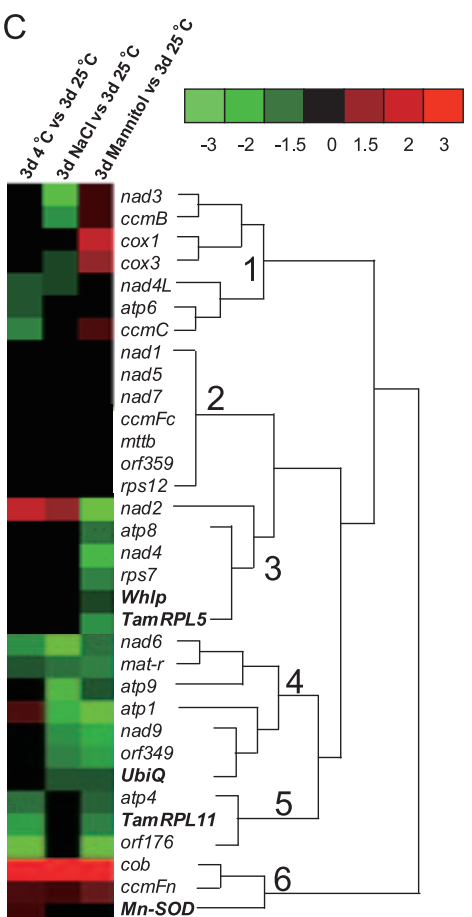

Fig. 4. Hierarchical cluster analysis of mitochondrial transcripts in germinating embryos and seedlings under different abiotic stress conditions. Actin transcript was used as a between array control. (A) Low temperature stress for 1, 2 and 3 days vs normal condition. (B) Low temperature stress for 1 day at the first, second and third days vs normal condition. (C) Low temperature, high salinity and osmotic stresses for the continuous 3 days vs normal condition. Green indicates 1- to 3-fold decreases, black constant (0; no significant change) and red 1- to 3-fold increases in the steady state mRNA abundance as compared to that under the normal condition at $25^{\circ} \mathrm{C}$ at the same time periods. Arabic letters indicate clusters identified by the clustering program. Each transcript is represented by a single row of colored boxes, and each condition is represented by single column. Asterisks denote genes whose transcripts were not detected in dry seeds, but readily detected in germinating embryos and were arbitrary scored as 4 fold decrease. 
changes during the second day as compared with the first day (cluster 7 in Fig. 4A). Taken together, our results showed that during the first day of low temperature stress most of the genes showed decreases in their expression levels more than 1.5-fold, while after the 2-days and 3 -days stress most of the genes displayed no changes in their transcript abundance.

When the low temperature stress was applied for 1 day during the first, second and third day after germination more than a half (54\%) of the mitochondrial genes fell into two clusters showing continuous decreases in their transcript levels (clusters 1 and 2 in Fig. 4B). Five genes in cluster 3 showed increases during the third day of low temperature stress, while three nuclear encoded and mitochondria-targeted genes fell into cluster 4 showing down regulation during the first day with no changes thereafter. Transcript profiles were then compared in seedlings subjected to the 3-days continuous stresses including the salinity and high osmotic potential stresses under normal temperature (Fig. 4C). Although seedlings under these different stress conditions might not be at the same developmental stages, a striking similarity was manifested in the expression patterns of more than a half of the genes studied. All genes were grouped into 6 clusters when subjected to all three kinds of stresses for the same period of 3 days. Notably a greater similarity was observed in the mitochondrial gene expression patterns between low temperature and high salinity stress than between low temperature and high osmotic potential stress. Cluster 1 included genes that responded specifically to the different stresses applied. On the other hand, cluster 2 included genes whose transcript levels displayed no changes to the stresses applied, and cluster 3 included genes whose transcripts responded with decrease specifically to the high osmotic stress. Cluster 4 consisted of genes that showed continuous decreases in their transcript levels commonly under high salinity and high osmotic potential stresses, and cluster 5 consisted genes commonly expressed under low temperature and high osmotic potential stresses. Two genes in cluster 6 ( $c o b$ and $c c m F n$ ) showed increases in their transcript levels commonly under all stress conditions, while the transcript level of $M n S O D$ increased only under the low temperature stress.

Quantitative real-time RT-PCR analysis of selected mitochondrial transcripts To confirm the reliability of macroarray data, transcript levels of 8 selected genes spotted were analyzed by quantitative real-time RT-PCR (qRT-PCR) using independently prepared RNA from new samples. These included four genes ( $n a d 7, c o b, c o x 2$ and atp6) encoding subunits of the respiratory complexes, one gene encoding ribosomal protein ( $r p s 7)$ and three nuclearencoded mitochondria-targeted genes (MnSOD, TaMRPL5 and Waox1a). As a control we used an isogene of wheat actin (gi:48927617), which gave a fairly constant transcript level under all conditions tested. In the qRT-PCR analysis we used at least three independently prepared RNA samples. The observed transcript levels in the seedlings incubated under low temperature for 1 and 2 days (Fig. 5A) and ones incubated for 3 days under all three kinds of stresses (Fig. 5B) were compared with those under the normal control condition incubated for the same periods. The analysis revealed that the transcript level of nad7 (complex I) decreased by 1-day low temperature stress but it did not change under all other stress conditions, agreeing with the array results (Fig. 4). Transcript abundance of $c o b$ gene also decreased after 1-day low temperature stress but it increased under all other stress conditions, also agreeing with the array results. The observed change in the abundance of $c o b$ transcript was in contrast to the report by Howell et al.
A

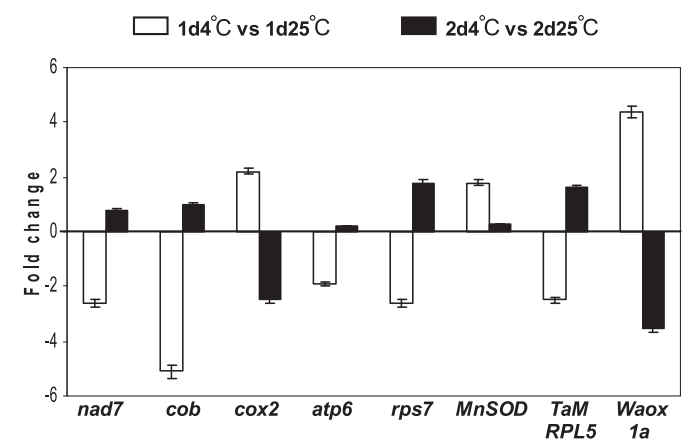

B

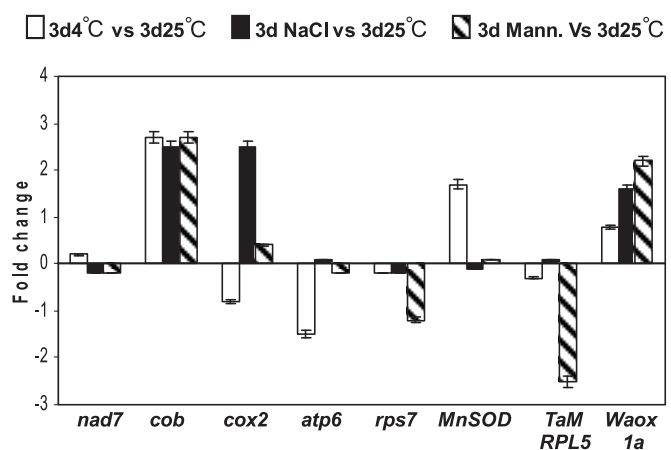

Fig. 5. Relative transcript abundance as estimated by qRT-PCR analysis. Transcript profiles of 5 genes encoding mitochondrial protein subunits and 3 nuclear encoded mitochondria-targeted genes were analyzed using samples incubated under the normal and stressed conditions. Each bar represents a mean \pm standard deviation from three independent experiments. For the stress conditions, see the legend to Fig. 1. 
(2007) in that the $c o b$ transcript was maintained at a similar level both under aerobic and anaerobic conditions in rice. The difference might be ascribed to the different stress conditions. The amount of atp6 transcript decreased after 1 and 3 days of low temperature stress but it remained fairly constant under 3-days salinity and osmotic stresses. The observed expression pattern of mitochondrially encoded ribosomal subunit gene, rps7, was similar to that of the nuclear-encoded mitochondriatargeted ribosomal subunit gene TaMRPL5. qRT-PCR also confirmed the increase in $M n S O D$ transcripts under 1 and 3 days of low temperature stress (Fig. 5) as estimated by the mitochondrial array (Fig. 4), although the transcript level did not show changes after the 2nd day of stress. Table 1 shows a good correlation between the macroarray data and qRT-PCR data, as an example, between the seedlings treated by low temperature for 1 day and non-treated control seedlings.

Table 1. Comparison of macroarray data and qRT-PCR data obtained for 6 genes in the stressed and non-stressed seedlings. A comparison represents an example made for 1 day $/ 25^{\circ} \mathrm{C}$ versus 1 day $/ 4^{\circ} \mathrm{C}$

\begin{tabular}{lcc}
\hline \hline \multicolumn{1}{c}{ Gene } & $\begin{array}{c}\text { Fold change } \\
\text { Macroarray }\end{array}$ & $\begin{array}{c}\text { Fold change } \\
\text { qRT-PCR }\end{array}$ \\
\hline nad7 & -2.1 & -2.6 \\
cob & -2.3 & -5.1 \\
cox2 & nd & 2.2 \\
atp6 & -2.0 & -1.9 \\
rps7 & -2.2 & -2.6 \\
MnSOD & -1.6 & -2.5 \\
TaMRPL5 & 2.2 & 1.8 \\
\hline
\end{tabular}

Figures represent means of two independent measurements. - indicates fold decreases in the stressed seedlings as compared to the normal seedlings. nd represents not repeatedly detected.

In our array experiment, cox2 and Waox1a, for unknown reason, could not be reproducibly detected. We therefore studied these transcripts by qRT-PCR. In wheat, cox 2 mRNA level was reported to be constant until 6 days after germination under normal condition (LiPook-Than et al., 2004). Under the stress conditions studied, however, abundance of cox2 transcript markedly increased during the first day of low temperature stress, while it decreased during the second and third days (Fig. 5). Under 3-days high salinity stress even a greater increase occurred in the amount of cox 2 transcript. Howell et al. (2007) reported that cox 2 was insensitive to oxygen until $12 \mathrm{~h}$ but then the level of its transcript increased by 3 -fold under aerobic condition compared with anaerobic condition. Transcript level of the wheat AOX gene, Waox1a, showed a more than four-fold increase after 1-day low temperature stress as compared to the normal condition but it decreased after the 2 nd day and remained unchanged until the 3rd day. Three-days stresses of high salinity and high osmotic potential stresses increased more of the Waox1a transcript than low temperature stress given for the same period.

\section{DISCUSSION}

Overall mitochondrial transcript profiles under stress conditions All of the 33 genes were categorized according to the results of the mitochondrial transcript abundance assay (Figs. 4 and 5). The relationships of gene expression levels are diagrammed in Fig. 6. The genes were grouped into 10 clusters according to five different stress conditions and their eight combinations of either two or three different stress conditions. In these clusters genes are redundantly grouped because of their similar or different responses to different stress conditions. According to this diagram, more genes showed decreases rather than increases in their transcript levels under the various stress conditions. The decreased gene expression agrees with the decreased growth (Fig. 1) but is in clear contrast to the increased respiration (Fig. 2) under the stress conditions. Respiration increases under stress conditions as compared to normal growth conditions (Stewart et al., 1990a; Vanlerberghe and McIntosh, 1992; Ribas-Carbo et al., 2005). These results suggest that the respiratory components in mitochondria remain functional under the imposed stresses during the early stage of wheat development. Mitochondrial protein levels are under control of post-trancriptional mRNA regulation and protein stability (Polosa and Attardi, 1991; Scarpulla, 2008). To gain further insight into mitochondrial biogenesis and functioning under abiotic stresses, changes in the abundance of mitochondrial protein components has to be studied and compared with changes in the mitochondrial transcriptome.

Expression dynamics of mitochondrial transcripts under abiotic stresses The observation that more than a half of the transcripts were down-regulated during the first and second day of low temperature stress (Figs. $4 \mathrm{~A}$ and $4 \mathrm{~B}$ ) indicated that mitochondrial gene expression was generally depressed under low temperature as to prevent further germination and to postpone the developmental program until favorable conditions prevail. By contrast, respiration increased dramatically under these conditions (Fig. 2). This negative relationship between the increased respiration and the decreased gene expression likely suggests that nuclear signals act to down-regulate the mitochondrial transcriptional machinery without suppressing the respiratory activity. Such suppressing effect of low temperature on mitochondrial gene expression was most pronounced at the earliest stage of germination. It is also notable that five of the down-regulated genes belong to the ones encoding respiratory com- 
A

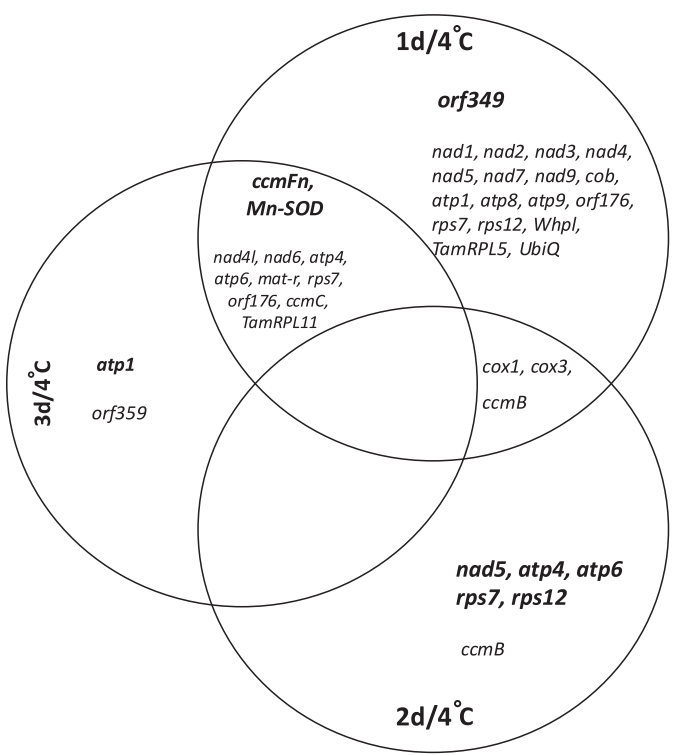

B

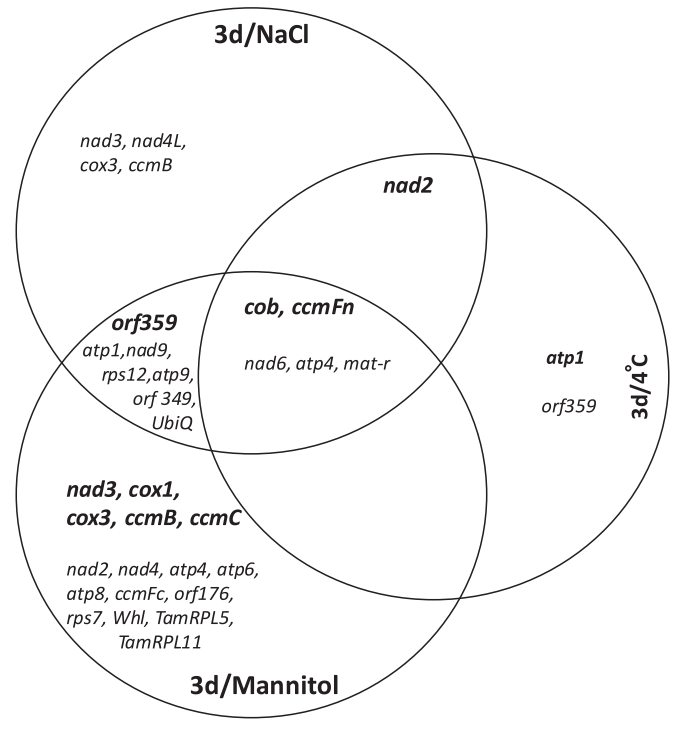

Fig. 6. Venn diagram showing the distribution of genes exhibiting common and specific responses to different stresses. Genes in bold letters indicate ones up-regulated and those in normal letters down-regulated under each stress condition as compared to the normal condition. Only genes showing more than 1.5 -fold change in their transcript levels are presented. For the stress conditions, see the legend to Fig. 1.

plex I subunits. This indicates that down regulation of complex I in the reparatory pathway might be an adaptive response during the early stages of wheat development to low temperature stress.

It was noted that the amount of transcripts of all nuclear-encoded and mitochondria-targeted genes showed decreases or stayed constant except for MnSOD transcript that showed increases in its abundance under the low temperature stress (Figs. 4C and 5). Expression of wheat $M n S O D$ was reported to increase following exposure to low temperature (Baek and Skinner, 2003, 2006). Mitochondrial electron transport is implicated in the production of reactive oxygen species during different biotic and abiotic stresses (Moller, 2001; Navrot et al., 2007). $M n S O D$ when over-expressed enhanced tolerance to chilling stress in Chlorella ellipsoidea (Clare et al., 1984), Zea mays (Van Breusegem et al., 1999), tobacco (Bowler et al., 1992; McKersie et al., 1993) and Brassica (Gachon et al., 2004). On the other hand, down regulation of the other nuclear encoded genes indicates that nuclear transcripts become limiting factors for the synthesis and assembly of functional mitochondrial complexes under the stress conditions.

Transcript level of the wheat AOX gene, Waox1a, showed a more than four-fold increase after 1-day low temperature stress as compared to the normal condition but it decreased after the 2nd day and remained unchanged thereafter. Three-days stresses of high salinity and osmotic potential increased more of the Waox1a transcript than low temperature stress given for the same period. Clifton et al. (2005) observed that components of the phosphorylating respiratory chain remained largely at constant levels but AOX responded differentially when Arabidopsis cell culture was subjected to a broad range of abiotic stresses and different types of physicochemical treatment. When grown at low temperature $\left(4^{\circ} \mathrm{C}\right)$ the alternative pathway increased its contribution to the total respiration in whole tissues and/or in isolated mitochondria in wheat (McCalg and Hill, 1977; Mizuno et al., 2008), potato (Hemrika-Wanger et al., 1983), maize (Stewart et al., 1990a, 1990b), Brassica (Rychter et al., 1988) and cucumber (Ordentlich et al., 1991). Drought stress increased the amount of leaf AOX protein and enhanced the rate of AOX dependent $\mathrm{O}_{2}$ uptake in wheat (Bartoli et al., 2005). These results suggest a role of AOX as scavenger of reactive oxygen species under stresses.

We also compared the expression patterns of wheat mitochondrial genes transcribed in polycistronic units. For the three genes belonging to the polycistronic unit of nad3-rps12-atp8 (Gualberto et al., 1991; Kitagawa et al., 2003), we found similar and different expression patterns among the individual members under different stress conditions (Fig. 4). A similar situation was found also under normal conditions (Khanam et al., 2007). With respect to another polycistronic unit of nad6-nad1 in wheat (Haouazine et al., 1993), nad6 transcript showed decreases by all three stresses but nad1 transcript decreased mainly by low temperature stress. These prob- 
ably imply a post-transcriptional control mechanism that is especially activated during stress conditions (Cahoon et al., 2008).

A number of genes showed osmotic stress specific responses with either up- or down-regulation, while four and two genes showed high salinity and low temperature stress specific responses, respectively, after 3-days stresses (Fig. 6). On the other hand, 5 genes showed common responses to the all stresses, among which cob and $c c m F n$ commonly increased their transcript levels but transcripts of other genes (nad6, atp4 and mat-r) decreased. In Arabidopsis, cold, salt and high osmotic potential stresses either commonly or differentially affect nuclear gene expression (Kreps et al., 2002) and a majority of changes are specific to the particular stress applied (Seki et al., 2002). Also in drought and salinity stressed barley (Hordeum vulgare), a majority of changes in nuclear encoded transcripts appeared to be stress specific (Ozturk et al., 2002). Our results suggest that changes in the mitochondrial gene expression profiles caused by different stresses also are mainly stress-specific, and thus individual genes respond differently to different stresses.

A higher similarity was observed in the gene expression profiles between salinity and osmotic stresses ( 7 genes) than between low temperature and high salinity (1 gene), while 5 genes were commonly regulated. A higher similarity in signaling pathways between drought and high salinity than between low temperature and high salinity was reported based on the nuclear gene expression in rice (Rabbani et al., 2003). These results indicate the existence of common and different regulatory systems that can sense different stresses and modulate not only nuclear but also mitochondrial gene expression. To further elucidate the role of mitochondria in stress responses, unique changes in mitochondrial transcriptome need to be identified by comparing cold/salinity/osmotic stress tolerant and sensitive lines. Because plants respond differently to the same stresses at different developmental stages, effects of developmental stages on the mitochondrial transcriptome also need to be studied. For such a study in wheat, mitochondrial array should provide a powerful tool if combined with nuclear transcriptome analysis (Kawaura et al., 2006). Array results combined with proteomic data should lead to the identification of a set of nuclear genes encoding mitochondrial multiprotein complexes.

The work was supported in part by a grant-in-aid from the Ministry of Education, Culture, Sports, Science and Technology of Japan (to C. N., no. 13306002). N. G. N. and M. S. were recipients of Japanese Society for the Promotion of Science Postdoctoral Fellowship (grant no. P05187 and P06193, respectively) and S. K. was a recipient of Japanese Ministry of Education Predoctoral Scholarship.

\section{REFERENCES}

Almansouri, M., Kinet, J. M., and Lutts, S. (2001) Effect of salt and osmotic stresses on germination in durum wheat (Triticum durum Desf.). Plant and Soil 231, 243-254.

Baek, K-H., and Skinner, D. Z. (2003) Alternation of antioxidant enzyme gene expression during cold acclimation of near isogenic wheat lines. Plant Science 165, 1221-1227.

Baek, K-H, and Skinner, D. Z. (2006) Differential expression of manganese superoxide dismutase sequence variants in near isogenic lines of wheat during cold acclimation. Plant Cell Rep. 25, 223-230.

Bartoli, C. G., Gomez, F., Gergoff, G., Guiamet, J. J., and Puntarulo, S. (2005) Up-regulation of the mitochondrial alternative oxidase pathway enhances photosynthetic electron transport under drought conditions. J. Exp. Bot. 56 1269-1276.

Bohnert, H. J., Nelson, E. D., and Jensen, R. G. (1995) Adaptation to environmental stresses. Plant Cell 7, 1099-1111.

Bowler, C., Montagu, M. V., and Inze, D. (1992) Superoxide dimutase and stress tolerance. Annu. Rev. Plant Physiol. Mol. Biol. 43, 83-116.

Bray, E. A., Bailey-Serres, J., and Weretilnyk, E. (2000) Responses to abiotic stresses, in: W. Gruissem, B. Buchannan, R. Jones (Eds.), Biochem. Mol. Biol. of Plants pp. 158-1249.

Cahoon, A. B., Takacs, E. M., Sharpe, R. M., and Stern, D. B. (2008) Nuclear, chloroplast, and mitochondrial transcript abundance along a maize leaf developmental gradient. Plant Mol. Biol. 66, 33-46.

Clare, D. A., Rabinowitch, H. D., and Fridovich, I. (1984) Superoxide dimutase and chilling injury in Chlorella ellipsoidea. Arch. Biochem. Biophys. 231, 158-168.

Clifton, R., Lister, R., Parker, K. L., Sappl, P. G., Elhafez, D., Millar, A. H., Day, D. A., and James, W. (2005) Stressinduced co-expression of alternative respiratory chain components in Arabidopsis thaliana. Plant Mol. Biol. 58, 193212.

de Hoon, M. J., Imoto, S., Nolan, J., and Miyano, S. (2004) Open source clustering software. Bioinformatics 20, 1453-1454.

Gachon, C., Mingam, A., and Charrier, B. (2004) Real-time PCR: What relevance to plant studies? J. Exp. Bot. 55, 1445-1454.

Giegé, P., Sweetlove, L. J., Cognat, V., and Leaver, C. J. (2005) Coordination of nuclear and mitochondrial genome expression during mitochondrial biogenesis in Arabidopsis. Plant Cell 17, 1497-1512.

Gualberto, J. M., Bonnard, G., Lamattina, L., and Grienenberger, J. M. (1991) Expression of the wheat mitochondrial nad3rps12 transcription unit: correlation between editing and mRNA maturation. Plant Cell 3, 1109-1120.

Haouazine, N., Takvorian, A., Jubier, M. F., Michel, F., and Lejeune, B. (1993) The nad6 gene and the exon d of nad1 are co-transcribed in wheat mitochondria. Curr. Genetics 24, 533-538.

Hemrika-Wanger, A. M., Verschoor, E. J., and Van der Plas, L. H. W. (1983) Alternative pathway respiration in vivo in potato tuber callus grown at various temperatures. Physiol. Plant 59, 369-374.

Hirai, M. S., Fujiwara, T., Awazuhara, M., Kimura. T., Noji, M., and Saito, K. (2003) Global expression profiling of sulfurstarved Arabidopsis by DNA macroarray reveals the role of $O$-acetyl-L-serine as a general regulator of gene expression in response to sulfur nutrition. Plant J. 33, 651-663.

Houde, M., Belcaid, M., Ouellet, F., Danyluk, J. F., Monroy, A., Dryanova, A., Gulick, P., Bergeron, A., Laroche, A., Links, M. G., et al. (2006) Wheat EST resources for functional genomics of abiotic stress. BMC Genomics 7, 149-170. 
Howell, K., Cheng, K., Murcha, M. W., Jenkin, L. E., Millar, A. H., and Whelan, J. (2007) Oxygen initiation of respiration and mitochondrial biogenesis in rice. J. Biol. Chem. 282, 15619-15631.

Kawasaki, S., Borchert, C., Deyholos, M., Wang, H., Brazile, S., Kawai, K., Galbrait, D., and Bohnert, H. (2001) Gene expression profiles during the initial phase of salt stress in rice. Plant Cell 13, 889-905.

Kawaura, K., Mochida, K., Yamazaki, Y., and Ogihara, Y. (2006) Transcriptome analysis of salinity stress responses in common wheat using a $22 \mathrm{k}$ oligo-DNA microarray. Funct. Integr. Genomics 6, 132-142.

Khanam, S., Naydenov, N. G., Kadowaki, K.-I., and Nakamura, C. (2007) Mitochondrial biogenesis as revealed by mitochondrial transcript profiles during germination and early seedling growth in wheat. Genes Genet. Syst. 82, 409-420.

Kitagawa, K., Takumi, S., and Nakamura, C. (2003) Selective transcription and post-transcriptional processing of the heteroplasmic mitochondrial orf156 copies in the nucleus-cytoplasm hybrids of wheat. Plant Mol. Biol. 53, 609-619.

Kreps, J. A., Wu, Y., Chang, H.-S., Zhu, T., Wang, X., and Harper, J. F. (2002) Transcriptome changes for Arabidopsis in response to salt, osmotic and cold stress. Plant Physiol. 130, 2129-2141.

Li-Pook-Than, J., Carrillo, C., and Bonen, L. (2004) Variation in mitochondrial transcript profiles of protein-coding genes during early germination and seedling development in wheat. Curr. Genet. 46, 374-380.

Livak, K. J., and Schmittgen, T. D. (2001) Analysis of relative gene expression data using real-time quantitative PCR and the $2^{-\Delta \Delta \mathrm{CT}}$ Method. Methods 25, 402-408.

Livne, A., and Livne, N. (1967) Tissue respiration and mitochondrial oxidative phosphorylation of $\mathrm{NaCl}$-treated pea seedlings. Plant Physiol. 42, 407-414.

Macherel, D., Benamer, A., Avelange-Macherel, M.-H., and Tolleter, D. (2007) Function and stress tolerance of seed mitochondria. Physiol. Plant 129, 233-241.

McCalg, T. N., and Hill, R. E. (1977) Cyanide-insensitive respiration in wheat: cultivar differences and effects of temperature, carbon dioxide, and oxygen. Can. J. Bot. 55, 549-555.

McKersie, B. D., Chen, Y., De Beus, M., Bowley, S. R., Bowler, C., Inze, D., D'Halluin, K., and Botterman, J. (1993) Superoxide dismutase enhances tolerance of freezing stress in transgenic alfalfa (Medicago sativa L.). Plant Physiol. 103, $1155-1163$.

Mizuno, N., Sugie, A., Kobayashi, F., and Takumi, S. (2008) Mitochondrial alternative pathway is associated with development of freezing tolerance in common wheat. J. Plant Physiol. 165, 462-467.

Moller, I. M. (2001) Plant mitochondria and oxidative stress: electron transport, NADPH turnover, and metabolism of reactive oxygen species. Ann. Rev. Plant Physiol. Plant Mol. Biol. 52, 561-591.

Morohashi, Y. (1986) Patterns of mitochondrial development in reserve tissues of germinated seeds: a survey. Physiol. Plant 66, 653-658.

Navrot, N., Rouhier, N., Gelhaye, E., and Jacquot, J. P. (2007) Reactive oxygen species generation and antioxidant systems in plant mitochondria. Physiol. Plant 129, 185-195.

Naydenov, G. N., Khanam, S. M., Atanassov, A., and Nakamura, C. (2008) Expression profiles of respiratory components associated with mitochondrial biogenesis during germination and seedling growth under normal and restricted conditions in wheat. Genes Genet. Syst. 83, 31-41.

Nieman, R. H. (1962) Some effect of sodium chloride on growth, photosynthesis and respiration of twelve crop plants. Botanical Gazet 123, 279-285.

Notsu, Y., Masood, S., Nishikawa, T., Kubo, N., Akiduki, G., Nakazono, M., Hirai, A., and Kadowaki, K. (2002) The complete sequence of the rice (Oryza sativa L.) mitochondrial genome: frequent DNA sequence acquisition and loss during the evolution of flowering plants. Mol. Genet. Genomics 268, 434-445.

Ogihara, Y., Yamazaki, Y., Murai, K., Kanno, A., Terachi, T., Shiina, T., Miyashita, N., Nasuda, S., Nakamura, C., Mori, N., et al. (2005) Structural dynamics of cereal mitochondrial genomes as revealed by complete nucleotide sequencing of the wheat mitochondrial genome. Nucl. Acid Res. 33, $6235-6250$

Ordentlich, A., Linzer, R. A., and Raskin, I. (1991) Alternative respiration and heat evolution in plants. Plant Physiol. 97, $1545-1550$.

Ozturk, Z. N., Talame, V., Deyholos, M., Michalowski, C. B., Galbraith, D. W., Gozukirmizi, N., Tuberosa, R., and Bohnert, H. J. (2002) Monitoring large scale changes in transcript abundance in drought- and salt- stressed barley. Plant Mol. Biol. 48, 551-573.

Polosa, P. L., and Attardi, G. (1991) Distinctive pattern and translational control of mitochondrial protein synthesis in rat brain synaptic endings. J. Biol. Chem. 266, 1001110017.

Rabbani, M. A., Maruyama, K., Abe, H., Khan, A., Katsura, K., Ito, Y., Yoshowara, K., Seki, M., Shinozaki, K., and Yamaguchi-Shinozaki, K. (2003) Monitoring expression profiles of rice genes under cold, drought and high salinity stresses and abscisic acid application using cDNA microar ray and RNA gel blot analyses. Plant Physiol. 133, 17551767.

Rachmilevitch, R., Xu, Y., Gonzalez-Meler, M. A., Huang, B. R., and Lambers, H. (2007) Cytochrome and alternative pathway activity in roots of thermal and non-thermal Agrostis species in response to high soil temperature. Physiol. Plant 129, 163-174.

Ribas-Carbo, M., Taylor, N. L., Giles, L., Busquets, S., Finnegan, P. M., Day, D. A., Lambers, H., Medrano, H., Berry, J. A., and Flexas, J. (2005) Effects of water stress on respiration in soybean leaves. Plant Physiol. 139, 466-473.

Rychter, A. M., Ciesla, E., and Kacperska, A. (1988) Participation of the cyanide-resistant pathway in respiration of winter rape leaves is affected by plant cold acclimation. Physiol. Plant. 73, 299-304.

Saldanha, A. J. (2004) Java Treeview-extensible visualization of microarray data. Bioinformatics 20, 3246-3248.

Scarpulla, R. C. (2008) Transcriptional paradigms in mammalian mitochondrial biogenesis and function. Physiol. Rev. 88, 611-638.

Seki, M., Narusaka, M., Ishida, J., Nanjo, T., Fujita, M., Oono, Y., Kamiya, A., Nakajima, M., Enju, A., Sakurai, T., et al. (2002) Monitoring the expression profiles of 7000 Arabidopsis genes under drought, cold and high salinity stresses using a full-length cDNA microarray. Plant J. 31, 279-292.

Shinozaki, K., Shinozaki, K. Y., and Seki, M. (2003) Regulatory network of gene expression in the drought and cold stress responses. Curr. Opin. Plant Biol. 6, 410-417.

Stewart, C. R., Martin, B. A., Reding, L., and Cerwick, S. (1990a) Respiration and alternative oxidase in corn seedling tissue during germination at different temperatures. Plant Physiol. 92, 755-760.

Stewart, C. R., Martin, B. A., Reding, L., and Cerwick, S. (1990b) Seedling growth, mitochondrial characteristics and 
alternative respiratory capacity of corn genotypes differing in cold tolerance. Plant Physiol. 92, 761-766.

Takumi, S., Tomioka, M., Eto, K., Naydenov, N., and Nakamura, C. (2002) Characterization of two non-homoeologous nuclear genes encoding mitochondrial alternative oxidase in common wheat. Genes Genet. Syst. 77, 81-88.

Talame, V., Ozturk, N. Z., Bohnert, H. J., and Roberto, T. (2007) Barley transcript profiles under dehydration shock and drought treatments: a comparative analysis. J. Exp. Bot. $\mathbf{5 8}, 229-240$.

Taylor, N. L., Heazlewood, J. H., Day, D. A., and Millar, A. H. (2005) Differential impact of environmental stresses on the pea mitochondrial proteome. Mol. Cell Proteomics 4, 11221133.

Thomashow, M. F. (1999) Plant cold acclimation: freezing tolerance genes and regulatory mechanisms. Ann. Rev. Plant
Physiol. Plant Mol. Biol. 50, 571-599.

Van Breusegem, F., Slooten, L., Stassart, J. M., Botterman, J., Moens, T., van Montagu, M., and Inze, D. (1999) Effects of overproduction of tobacco MnSOD in maize chloroplast on foliar tolerance to cold and oxidative stress. J. Exp. Bot. 50, $1-78$.

Vanlerberghe, G. C., and McIntosh, L. (1992) Lower growth temperature increases alternative pathway capacity and alternative oxidase protein in tobacco. Plant Physiol. 100, 115119.

Zheng, J., Zhao, J., Tao, Y., Wang, J., Liu, Y., Junjie, F., Jin, Y., Gao, P., Zhang, J., Bai, Y., and Wang, G. (2004) Isolation and analysis of water stress induced genes in maize seedlings by subtractive PCR and cDNA macroarray. Plant Mol. Biol. 55, 807-823. 